\title{
Boundedness of Marcinkiewicz integrals with mixed homogeneity along compound surfaces
}

Daiqing Zhang ${ }^{1 *}$ and Feng Liu²

\section{"Correspondence:}

zhangdaiqing2011@163.com

${ }^{1}$ School of Mathematical Sciences,

Xiamen University, Xiamen, 361005,

China

Full list of author information is

available at the end of the article

\begin{abstract}
In this note we establish the $L^{p}$ boundedness of Marcinkiewicz integrals with mixed homogeneity along compound surfaces, which improve and extend some previous results. The main ingredient is to present a systematic treatment with several singular integral operators.
\end{abstract}

MSC: 42B20; 42B15; 42B25

Keywords: Marcinkiewicz integrals; rough kernels; mixed homogeneity; Littlewood-Paley theory

\section{Introduction}

Let $\mathbb{R}^{n}, n \geq 2$, be the $n$-dimensional Euclidean space and $S^{n-1}$ denote the unit sphere in $\mathbb{R}^{n}$ equipped with the induced Lebesgue measure $d \sigma$. Let $\alpha_{j} \geq 1(j=1, \ldots, n)$ be fixed real numbers. Define the function $F: \mathbb{R}^{n} \times(0, \infty) \longrightarrow \mathbb{R}$ by $F(x, \rho)=\sum_{j=1}^{n} x_{j}^{2} \rho^{-2 \alpha_{j}}, x=\left(x_{1}, x_{2}, \ldots, x_{n}\right)$. It is clear that, for each fixed $x \in \mathbb{R}^{n}$, the function $F(x, \rho)$ is a decreasing function in $\rho>0$. We let $\rho(x)$ denote the unique solution of the equation $F(x, \rho)=1$. Fabes and Rivière [1] showed that $\left(\mathbb{R}^{n}, \rho\right)$ is a metric space, which is often called the mixed homogeneity space related to $\left\{\alpha_{j}\right\}_{j=1}^{n}$. For $\lambda>0$, we let $A_{\lambda}$ be the diagonal $n \times n$ matrix $A_{\lambda}=\operatorname{diag}\left\{\lambda^{\alpha_{1}}, \ldots, \lambda^{\alpha_{n}}\right\}$. Let $\mathbb{R}^{+}:=(0, \infty)$ and $\varphi: \mathbb{R}^{+} \rightarrow \mathbb{R}^{+}$, we denote $A_{\varphi(\rho(y)) y^{\prime}}$ by $A_{\varphi}(y)$ for $y \in \mathbb{R}^{n}$, where $y^{\prime}=A_{\rho(y)^{-1}} y \in S^{n-1}$.

The change of variables related to the spaces $\left(\mathbb{R}^{n}, \rho\right)$ is given by the transformation

$$
\begin{aligned}
& x_{1}=\rho^{\alpha_{1}} \cos \theta_{1} \cdots \cos \theta_{n-2} \cos \theta_{n-1}, \\
& x_{2}=\rho^{\alpha_{2}} \cos \theta_{1} \cdots \cos \theta_{n-2} \sin \theta_{n-1}, \\
& \cdots, \\
& x_{n-1}=\rho^{\alpha_{n-1}} \cos \theta_{1} \sin \theta_{2}, \\
& x_{n}=\rho^{\alpha_{n}} \sin \theta_{1} .
\end{aligned}
$$

Thus $d x=\rho^{\alpha-1} J\left(x^{\prime}\right) d \rho d \sigma\left(x^{\prime}\right)$, where $\rho^{\alpha-1} J\left(x^{\prime}\right)$ is the Jacobian of the above transform and $\alpha=\sum_{j=1}^{n} \alpha_{j}, J\left(x^{\prime}\right)=\sum_{j=1}^{n} \alpha_{j}\left(x_{j}^{\prime}\right)^{2}$. Obviously, $J\left(x^{\prime}\right) \in \mathcal{C}^{\infty}\left(S^{n-1}\right)$ and there exists $M>0$ such 
that

$$
1 \leq J\left(x^{\prime}\right) \leq M, \quad \forall x^{\prime} \in S^{n-1}
$$

It is easy to see that

$$
\rho(x)=|x|, \quad \text { if } \alpha_{1}=\alpha_{2}=\cdots=\alpha_{n}=1 .
$$

Let $\Omega$ be integrable on $S^{n-1}$ and satisfy

$$
\begin{aligned}
& \int_{S^{n-1}} \Omega(u) J(u) d \sigma(u)=0, \\
& \Omega\left(A_{s} x\right)=\Omega(x), \quad \forall s>0 \text { and } x \in \mathbb{R}^{n} .
\end{aligned}
$$

For $d \geq 2$ and a suitable function $\Phi: \mathbb{R}^{n} \rightarrow \mathbb{R}^{d}$, we define the parabolic Marcinkiewicz integral operator $\mathcal{M}_{\Omega}^{\Phi}$ on $\mathbb{R}^{d}$ by

$$
\mathcal{M}_{\Omega}^{\Phi}(f)(x)=\left(\int_{0}^{\infty}\left|\frac{1}{t} \int_{\rho(y) \leq t} \frac{\Omega(y)}{\rho(y)^{\alpha-1}} f(x-\Phi(y)) d y\right|^{2} \frac{d t}{t}\right)^{1 / 2} .
$$

When $\alpha_{1}=\cdots=\alpha_{n}=1$, we denote $\mathcal{M}_{\Omega}^{\Phi}$ by $\mu_{\Omega}^{\Phi}$. Clearly, if $n=d$ and $\Phi(y)=y$, the operator $\mu_{\Omega}^{\Phi}$ reduces to the classical Marcinkiewicz integral operator denoted by $\mu_{\Omega}$, which was introduced by Stein [2] and investigated by many authors (see [3-9] for example). In particular, Ding et al. [5] proved that if $\Omega \in H^{1}\left(S^{n-1}\right)$, then $\mu_{\Omega}$ is bounded on $L^{p}\left(\mathbb{R}^{n}\right)$ for $1<p<\infty$. Subsequently, Chen et al. [4] showed that $\mu_{\Omega}$ is bounded on $L^{p}\left(\mathbb{R}^{n}\right)$ for $2 \beta /(2 \beta-1)<p<2 \beta$ if $\Omega \in \mathcal{F}_{\beta}\left(S^{n-1}\right)$ for some $\beta>1$. Here

$$
\begin{aligned}
& \mathcal{F}_{\beta}\left(S^{n-1}\right) \\
& \quad:=\left\{\Omega \in L^{1}\left(S^{n-1}\right): \sup _{\xi \in S^{n-1}} \int_{S^{n-1}}\left|\Omega\left(y^{\prime}\right)\right|\left(\log \frac{1}{\left|\xi \cdot y^{\prime}\right|}\right)^{\beta} d \sigma\left(y^{\prime}\right)<\infty\right\}, \quad \forall \beta>0 .
\end{aligned}
$$

The functions class $\mathcal{F}_{\beta}\left(S^{n-1}\right)$ was introduced by Grafakos and Stefanov [10] in the study of $L^{p}$ boundedness of singular integral operator with rough kernels. It follows from [10] that $\mathcal{F}_{\beta_{1}}\left(S^{n-1}\right) \subsetneq \mathcal{F}_{\beta_{2}}\left(S^{n-1}\right)$ for $0<\beta_{2}<\beta_{1}$, and $\bigcup_{q>1} L^{q}\left(S^{n-1}\right) \subsetneq \mathcal{F}_{\beta}\left(S^{n-1}\right)$ for any $\beta>0$. Moreover,

$$
\bigcap_{\beta>1} \mathcal{F}_{\beta}\left(S^{n-1}\right) \nsubseteq H^{1}\left(S^{n-1}\right) \nsubseteq \bigcup_{\beta>1} \mathcal{F}_{\beta}\left(S^{n-1}\right)
$$

and

$$
\bigcap_{\beta>1} \mathcal{F}_{\beta}\left(S^{n-1}\right) \nsubseteq L \log ^{+} L\left(S^{n-1}\right)
$$

Later on, Al-Salman et al. [11] proved that $\mu_{\Omega}$ is bounded on $L^{p}\left(\mathbb{R}^{n}\right)$ for $1<p<\infty$ provided that $\Omega \in L\left(\log ^{+} L\right)^{1 / 2}\left(S^{n-1}\right)$. It is well known that $L\left(\log ^{+} L\right)^{1 / 2}\left(S^{n-1}\right)$ and $H^{1}\left(S^{n-1}\right)$ do not contain each other. When $n=d$ and $\Phi(y)=P(|y|) y^{\prime}$ with $P(y)$ being a real polynomial on $\mathbb{R}$ sat- 
isfying $P(0)=0$, Wu [12] proved that $\mu_{\Omega}^{\Phi}$ is bounded on $L^{p}\left(\mathbb{R}^{n}\right)$ for $1+1 /(2 \beta)<p<1+2 \beta$ provided that $\Omega \in \mathcal{F}_{\beta}\left(S^{n-1}\right)$ for some $\beta>1 / 2$. The $L^{p}$ boundedness for the Marcinkiewicz integral operator associated to polynomial mappings has also been obtained (see $[6,13])$. When $\alpha_{j} \geq 1(j=1, \ldots, n), n=d$ and $\Phi(y)=y$, we denote $\mathcal{M}_{\Omega}^{\Phi}$ by $\mathcal{M}_{\Omega}$. In 2008, Ding et al. [14] proved that $\mathcal{M}_{\Omega}$ is bounded on $L^{p}\left(\mathbb{R}^{n}\right)$ for $1<p<\infty$, provided that $\Omega \in L^{q}\left(S^{n-1}\right)$ for fixed $q>1$. Chen and Ding [15] extended the above result to the case $\Omega \in L(\log L)^{1 / 2}\left(S^{n-1}\right)$. Later on, Chen and Lu [16] proved that $\mathcal{M}_{\Omega}$ is bounded on $L^{p}\left(\mathbb{R}^{n}\right)$ for $2 \beta /(2 \beta-1)<p<2 \beta$, provided that $\Omega \in \mathcal{F}_{\beta}\left(S^{n-1}\right)$ for some $\beta>1$. This result was recently refined by Liu and $\mathrm{Wu}$ [17], who extended the range of $\beta$ to the case $\beta>1 / 2$ and the range of $p$ to the case $1+1 /(2 \beta)<p<1+2 \beta$. When $n=d$ and $\Phi(y)=A_{\varphi}(y)$, Al-Salman [18] obtained the following result.

Theorem A Let $n=d$ and $\Phi(y)=A_{\varphi}(y)$. Suppose that $\Omega \in \mathcal{F}_{\beta}\left(S^{n-1}\right)$ for some $\beta>1$ with satisfying (1.1)-(1.2).

(i) If $\varphi(t)=P(t)$ with $P$ being a real polynomial on $\mathbb{R}$, then $\mathcal{M}_{\Omega}^{\Phi}$ are bounded on $L^{p}\left(\mathbb{R}^{n}\right)$ for $2 \beta /(2 \beta-1)<p<2 \beta$. The bounds are independent of the coefficients of $P$.

(ii) If $\varphi \in \mathfrak{F}$, then $\mathcal{M}_{\Omega}^{\Phi}$ are bounded on $L^{p}\left(\mathbb{R}^{n}\right)$ for $2 \beta /(2 \beta-1)<p<2 \beta$. Here $\mathfrak{F}$ is the set of all functions $\phi$ which satisfy:

(a) $\phi: \mathbb{R}^{+} \rightarrow \mathbb{R}^{+}$is continuous increasing $\mathcal{C}^{1}$ function satisfying that $\phi^{\prime}$ is monotonous;

(b) there exist constant $C_{\phi}$ and $c_{\phi}$ such that $t \phi^{\prime}(t) \geq C_{\phi} \phi(t)$ and $\phi(2 t) \leq c_{\phi} \phi(t)$ for all $t>0$.

Remark 1.1 There are some model examples in the class $\mathfrak{F}$, such as $t^{\alpha}(\alpha>0), t^{\alpha}(\ln (1+t))^{\beta}$ $(\alpha, \beta>0), t \ln \ln (e+t)$, real-valued polynomials $P$ on $\mathbb{R}$ with positive coefficients and $P(0)=$ 0 and so on. For $\varphi \in \mathfrak{F}$, there exists a constant $B_{\varphi}>1$ such that $\varphi(2 t) \geq B_{\varphi} \varphi(t)$ (see [19]).

It is natural to ask whether Theorem A also holds if the range of $\beta$ is relaxed to $\beta>$ $1 / 2$ and the range of $p$ is relaxed to $1+1 /(2 \beta)<p<1+2 \beta$. In this paper, we will give an affirmative answer to this question. Our main results can be stated as follows.

Theorem 1.1 Let $n=d$ and $\Phi(y)=\left(P_{1}(\varphi(\rho(y))) y_{1}^{\prime}, \ldots, P_{n}(\varphi(\rho(y))) y_{n}^{\prime}\right)$ with $P_{i}(t)$ being real valued polynomials on $\mathbb{R}$ satisfying $P_{i}(0)=0$ and $\varphi \in \mathfrak{F}$. Suppose that $\Omega \in \mathcal{F}_{\beta}\left(S^{n-1}\right)$ for some $\beta>1 / 2$ satisfying (1.1)-(1.2). Then $\mathcal{M}_{\Omega}^{\Phi}$ are bounded on $L^{p}\left(\mathbb{R}^{n}\right)$ for $1+1 /(2 \beta)<p<$ $1+2 \beta$. The bounds are independent of the coefficients of $P_{j}$ for all $1 \leq j \leq n$ but depend on $\max _{1 \leq j \leq d} \operatorname{deg}\left(P_{j}\right)$ and $\varphi$.

Theorem 1.2 Let $n=d$ and $\Phi(y)=A_{P_{N}(\varphi)}(y)$ with $\varphi \in \mathfrak{F}$ and $P_{N}(t)=\sum_{i=1}^{N} a_{i} t^{i}$ and $P_{N}(t)>$ 0 if $t \neq 0$. Suppose that $\Omega \in \mathcal{F}_{\beta}\left(S^{n-1}\right)$ for some $\beta>1 / 2$ satisfying (1.1)-(1.2). Then $\mathcal{M}_{\Omega}^{\Phi}$ are bounded on $L^{p}\left(\mathbb{R}^{n}\right)$ for $1+1 /(2 \beta)<p<1+2 \beta$. The bounds are independent of the coefficients of $P_{N}$ but depend on $N$ and $\varphi$.

Remark 1.2 It is clear that Theorem 1.1 implies Theorem 1.2. When $\alpha_{1}=\cdots=\alpha_{n}=1$, $\varphi(t)=t$ and $P_{1}(t)=\cdots=P_{n}(t)=\sum_{i=1}^{N} a_{i} t^{i}$, Theorem 1.1 implies the result of [12]. In fact, Theorem 1.2 with $\varphi(t)=t$ extends the result of [12] to the mixed case. Comparing Theorem A with Theorem 1.2, the range of $\beta$ is extended to the case $\beta>1 / 2$ and the range of $p$ 
is enlarged to the case $1+1 /(2 \beta)<p<1+2 \beta$. Thus Theorem 1.2 essentially improves and generalizes the corresponding results in Theorem A. In addition, Theorem 1.2 implies the result [17, Theorem 1.3] when $P_{N}(t)=\varphi(t)=t$.

When $n=2$, we have the following result.

Theorem 1.3 Let $\phi=\left(\phi_{1}, \ldots, \phi_{d}\right)$ be real analytic on $S^{1}$. Let $\Phi(y)=P_{N}(\varphi(\rho(y))) \phi\left(y^{\prime}\right)=$ $\left(P_{N}(\varphi(\rho(y))) \phi_{1}\left(y^{\prime}\right), \ldots, P_{N}(\varphi(\rho(y))) \phi_{d}\left(y^{\prime}\right)\right)$ with $P_{N}(t)=\sum_{i=1}^{N} a_{i} t^{i}$ and $\varphi \in \mathfrak{F}$. Suppose that $\Omega \in \mathcal{F}_{\beta}\left(S^{1}\right)$ for some $\beta>1$ satisfying (1.1)-(1.2). Then $\mathcal{M}_{\Omega}^{\Phi}$ are bounded on $L^{p}\left(\mathbb{R}^{d}\right)$ for $1+1 /(2 \beta)<p<1+2 \beta$. The bounds are independent of the coefficients of $P_{N}$ but depend on $\varphi$ and $N$.

We remark that when $\alpha_{1}=\cdots=\alpha_{n}=1$ and $\varphi(t)=t$, the surface $\left\{\Phi(y): y \in \mathbb{R}^{n}\right\}$ given as in Theorem 1.3 recovers $\left\{\left(P_{N}(|y|) \phi_{1}\left(y^{\prime}\right), \ldots, P_{N}(|y|) \phi_{d}\left(y^{\prime}\right)\right) ; y \in \mathbb{R}^{n}\right\}$, which was originally introduced by Al-Balushi and Al-Salman [20] in the study of $L^{p}$ bounds of singular integrals associated to certain surfaces.

The third type of surfaces we consider are polynomial compound subvarieties. To state the rest of our result, we need to recall some notations. Let $\mathcal{A}(n, m)$ be the set of polynomials on $\mathbb{R}^{n}$ which have real coefficients and degrees not exceeding $m$, and let $V(n, m)$ be the collection of polynomials in $\mathcal{A}(n, m)$ which are homogeneous of degree $m$. For $P \in \mathcal{A}(n, m)$, we set

$$
\|P\|=\left\|\sum_{|\lambda| \leq m} a_{\lambda} y^{\lambda}\right\|=\left(\sum_{|\lambda| \leq m}\left|a_{\lambda}\right|^{2}\right)^{1 / 2} .
$$

Definition 1.1 ([21]) Let $n \geq 2, m \in \mathbb{N}$ and $\beta>0$. An integrable function $\Omega$ on $S^{n-1}$ is said to be in the space $\mathcal{F}(n, m, \beta)$ if

$$
\sup _{P \in V(n, m),\|P\|=1} \int_{S^{n-1}}|\Omega(y)|\left(\log ^{+} \frac{1}{|P(y)|}\right)^{\beta} d \sigma(y)<\infty
$$

It should be pointed out that the condition (1.5) was introduced by Al-Salman and Pan [21] (also see [22]) in a study of the $L^{p}$ boundedness of singular integrals with rough kernels. It is easy to check that $\mathcal{F}(n, 1, \beta)=\mathcal{F}_{\beta}\left(S^{n-1}\right)$. Moreover, it was shown in [21] that

$$
\mathcal{F}_{\beta}\left(S^{1}\right)=\bigcap_{m=1}^{\infty} \mathcal{F}(2, m, \beta) .
$$

The rest of the results can be stated as follows.

Theorem 1.4 Let $\mathcal{P}=\left(P_{1}, \ldots, P_{d}\right)$ with $P_{j}: \mathbb{R}^{n} \rightarrow \mathbb{R}$ being a polynomial for $1 \leq j \leq d$. Let $\Phi(y)=\mathcal{P}\left(\varphi(\rho(y)) y^{\prime}\right)$ and $\varphi \in \mathfrak{F}$. Suppose that $\Omega$ satisfies (1.1)-(1.2) and $\Omega \in \bigcap_{s=1}^{\infty} \mathcal{F}(n, s, \beta)$ for some $\beta>1 / 2$. Then $\mathcal{M}_{\Omega}^{\Phi}$ are bounded on $L^{p}\left(\mathbb{R}^{d}\right)$ for $1+1 /(2 \beta)<p<1+2 \beta$. The bounds are independent of the coefficients of $P_{j}$ for all $1 \leq j \leq d$ but depend on $\max _{1 \leq j \leq d} \operatorname{deg}\left(P_{j}\right)$ and $\varphi$.

Theorem 1.5 Let $\mathcal{P}=\left(P_{1}, \ldots, P_{d}\right)$ with $P_{j}: \mathbb{R}^{2} \rightarrow \mathbb{R}$ being a polynomial for $1 \leq j \leq d$. Let $\Phi(y)=\mathcal{P}\left(\varphi(\rho(y)) y^{\prime}\right)$ and $\varphi \in \mathfrak{F}$. Suppose that $\Omega$ satisfies (1.1)-(1.2) and $\Omega \in \mathcal{F}_{\beta}\left(S^{1}\right)$ for some 
$\beta>1 / 2$. Then $\mathcal{M}_{\Omega}^{\Phi}$ are bounded on $L^{p}\left(\mathbb{R}^{d}\right)$ for $1+1 /(2 \beta)<p<1+2 \beta$. The bounds are independent of the coefficients of $P_{j}$ for all $1 \leq j \leq d$ but depend on $\max _{1 \leq j \leq d} \operatorname{deg}\left(P_{j}\right)$ and $\varphi$.

Remark 1.3 When $\alpha_{j}=1(j=1, \ldots, n)$, Theorem 1.4 implies Theorem 1.2 with $\rho=1$ in [13]. Obviously, Theorem 1.5 follows from Theorem 1.4 because of (1.6).

The rest of this paper is organized as follows. After recalling some preliminary notations and lemmas in Section 2, we will prove our results in Section 3. We would like to remark that the main methods employed in this paper is a combination of ideas and arguments from $[12,21,23]$. The main ingredient in our proofs is to give a systematic treatment with these operators mentioned above.

Throughout this paper, we let $p^{\prime}$ satisfy $1 / p+1 / p^{\prime}=1$. The letter $C$, sometimes with additional parameters, will stand for positive constants, not necessarily the same one at each occurrence, but independent of the essential variables.

\section{Preliminaries}

Lemma 2.1 Let $\left\{\sigma_{j, t}\right\}$ be a family of measures. Suppose that

$$
\left\|\operatorname{supsup}_{j \in \mathbb{Z}}|| \sigma_{j, t}|* g|\right\|_{p} \leq C\|g\|_{p}
$$

holds for some $p>1$ and $g \in L^{p}\left(\mathbb{R}^{n}\right)$. Then there exists a constant $C>0$ such that

$$
\left\|\left(\int_{1}^{2} \sum_{j \in \mathbb{Z}}\left|\sigma_{j, t} * g_{j}\right|^{2} d t\right)^{1 / 2}\right\|_{p} \leq C\left\|\left(\sum_{j \in \mathbb{Z}}\left|g_{j}\right|^{2}\right)^{1 / 2}\right\|_{p}
$$

for arbitrary functions $\left\{g_{j}\right\}_{j \in \mathbb{Z}} \in L^{p}\left(\ell^{2}, \mathbb{R}^{n}\right)$.

Proof By the assumption, we have

$$
\left\|\sup _{j \in \mathbb{Z}} \sup _{t \in[1,2]}\left|\sigma_{j, t} * g_{j}\right|\right\|_{p} \leq\left\|\sup _{j \in \mathbb{Z}} \sup _{t>0}\left|\sigma_{j, t}\right| * \sup _{j \in \mathbb{Z}}\left|g_{j}\right|\right\|_{p} \leq C\left\|\sup _{j \in \mathbb{Z}}\left|g_{j}\right|\right\|_{p} .
$$

On the other hand, by the dual argument, there exists a function $h \in L^{p^{\prime}}\left(\mathbb{R}^{n}\right)$ satisfying $\|h\|_{p^{\prime}}=1$ such that

$$
\begin{aligned}
\left\|\int_{1}^{2} \sum_{j \in \mathbb{Z}}\left|\sigma_{j, t} * g_{j}\right| d t\right\|_{p} & =\int_{\mathbb{R}^{n}} \sum_{j \in \mathbb{Z}} \int_{1}^{2}\left|\sigma_{j, t} * g_{j}(x)\right| d t h(x) d x \\
& \leq \int_{\mathbb{R}^{n}} \sum_{j \in \mathbb{Z}} \int_{1}^{2}\left|\sigma_{j, t}\right| *\left|g_{j}(x)\right| d t|h(x)| d x \\
& \leq \int_{\mathbb{R}^{n}} \sum_{j \in \mathbb{Z}}\left|g_{j}(x)\right| \sup _{j \in \mathbb{Z}} \sup _{t \in[1,2]}\left|\sigma_{j, t}\right| *|\bar{h}|(x) d x \\
& \leq\left\|\sum_{j \in \mathbb{Z}}\left|g_{j}\right|\right\|_{p}\left\|\sup _{j \in \mathbb{Z}} \sup _{t>0}\left|\sigma_{j, t}\right| *|\bar{h}|(x)\right\|_{p^{\prime}} \\
& \leq C\left\|\sum_{j \in \mathbb{Z}}\left|g_{j}\right|\right\|_{p},
\end{aligned}
$$


where $\bar{h}(x)=h(-x)$. Thus, Lemma 2.1 follows from the standard interpolation arguments.

Let $\left\{a_{k}\right\}_{k \in \mathbb{Z}}$ be a sequence of real positive numbers with satisfying $\inf _{k \in \mathbb{Z}} a_{k+1} / a_{k}=a>1$. Let $\left\{\lambda_{k}\right\}_{k \in \mathbb{Z}}$ be a collection of $\mathcal{C}^{\infty}(0, \infty)$ functions satisfying the following conditions:

$$
\begin{aligned}
& \operatorname{supp}\left(\lambda_{k}\right) \subset\left[a_{k+1}^{-1}, a_{k-1}^{-1}\right] ; \\
& 0 \leq \lambda_{k} \leq 1 ; \quad \sum_{k \in \mathbb{Z}} \lambda_{k}^{2}(t)=1 ; \quad\left|d \lambda_{k}(t) / d t\right| \leq C / t,
\end{aligned}
$$

where $C$ is independent of $t$ and $k$. Let $M \in \mathbb{N} \backslash\{0\}$ and $L: \mathbb{R}^{n} \rightarrow \mathbb{R}^{M}$ be a linear transformation. For each $k \in \mathbb{Z}$, we define the multiplier operators $S_{k}$ in $\mathbb{R}^{n}$ by

$$
\widehat{S_{k} f}(\xi)=\lambda_{k}(|L(\xi)|) \hat{f}(\xi)
$$

By an argument which is similar to those used in [8, Proposition 3.1], one can easily get the following lemma. The details are omitted here.

Lemma 2.2 Let $S_{k}$ be as in (2.1) and $\left\{g_{j, k, t}\right\}$ arbitrary functions on $\mathbb{R}^{n}$. Then

(i) for each fixed $1<p<2$ and $1<q<p$,

$$
\left\|\left(\sum_{j \in \mathbb{Z}} \int_{1}^{2}\left|\sum_{k \in \mathbb{Z}} S_{j+k} g_{j, k, t}\right|^{2} d t\right)^{1 / 2}\right\|_{p}^{q} \leq C \sum_{k \in \mathbb{Z}}\left\|\left(\sum_{j \in \mathbb{Z}} \int_{1}^{2}\left|g_{j, k, t}\right|^{2} d t\right)^{1 / 2}\right\|_{p}^{q} ;
$$

(ii) for each fixed $2<p<\infty$ and $1<q<p^{\prime}$,

$$
\begin{aligned}
& \left\|\left(\sum_{j \in \mathbb{Z}} \int_{1}^{2}\left|\sum_{k \in \mathbb{Z}} S_{j+k} g_{j, k, t}\right|^{2} d t\right)^{1 / 2}\right\|_{p}^{q} \\
& \quad \leq C \sum_{k \in \mathbb{Z}}\left(\int_{1}^{2}\left\|\left(\sum_{j \in \mathbb{Z}}\left|g_{j, k, t}\right|^{2}\right)^{1 / 2}\right\|_{p}^{2} d t\right)^{q / 2} .
\end{aligned}
$$

The following lemma is our main ingredient in the proof of our main results.

Lemma 2.3 Let $\left\{\tau_{k, t}: k \in \mathbb{Z}, t \in \mathbb{R}^{+}\right\}$be a family of uniformly bounded Borel measures on $\mathbb{R}^{n}$. Let $\left\{a_{k}\right\}_{k \in \mathbb{Z}}$ be a sequence of real numbers with satisfying $\inf _{k \in \mathbb{Z}} a_{k+1} / a_{k}=a>1$. Let $M \in \mathbb{N} \backslash\{0\}$ and $L: \mathbb{R}^{n} \rightarrow \mathbb{R}^{M}$ be a linear transformation. Suppose that

$$
\begin{aligned}
& \text { (i) }\left|\widehat{\tau_{k, t}}(\xi)\right| \leq C \min \left\{1, a_{k}|L(\xi)|\right\} \text {; } \\
& \text { (ii) }\left|\widehat{\tau_{k, t}}(\xi)\right| \leq C\left(\log \left|a_{k} L(\xi)\right|\right)^{-\beta} \text { for some } \beta>0 \text {, if } a_{k}|L(\xi)|>1 \text {; } \\
& \text { (iii) }\left\|\sup _{k \in \mathbb{Z}} \sup _{t>0}|| \tau_{k, t}|* f|\right\|_{q} \leq C\|f\|_{q}
\end{aligned}
$$

for all $1<q<\infty$. Then for $p \in(1+1 /(2 \beta), 1+2 \beta)$ and $\beta>1 / 2$, there exists a constant $C(a)>0$ such that

$$
\left\|\left(\int_{1}^{2} \sum_{k \in \mathbb{Z}}\left|\tau_{k, t} * f\right|^{2} d t\right)^{1 / 2}\right\|_{p} \leq C(a)\|f\|_{p}
$$


Proof Let $S_{k}$ be as in (2.1). Then we can write

$$
\begin{aligned}
G(f)(x): & =\left(\int_{1}^{2} \sum_{k \in \mathbb{Z}}\left|\tau_{k, t} * f(x)\right|^{2} d t\right)^{1 / 2} \\
& =\left(\int_{1}^{2} \sum_{k \in \mathbb{Z}}\left|\tau_{k, t} *\left(\sum_{j \in \mathbb{Z}} S_{j+k} S_{j+k} f\right)(x)\right|^{2} d t\right)^{1 / 2} \\
& =\left(\sum_{k \in \mathbb{Z}} \int_{1}^{2}\left|\sum_{j \in \mathbb{Z}} S_{j+k}\left(\tau_{k, t} * S_{j+k} f\right)(x)\right|^{2} d t\right)^{1 / 2} .
\end{aligned}
$$

Case $1.1+1 /(2 \beta)<p<2$. It follows from (2.2) and (2.7) that

$$
\|G(f)\|_{p}^{q} \leq C \sum_{j \in \mathbb{Z}}\left\|\left(\sum_{k \in \mathbb{Z}} \int_{1}^{2}\left|\tau_{k, t} * S_{j+k} f\right|^{2} d t\right)^{1 / 2}\right\|_{p}^{q}, \quad \forall 1<q<p .
$$

For each fixed $k \in \mathbb{Z}$, we set

$$
I_{j} f(x):=\left(\sum_{k \in \mathbb{Z}} \int_{1}^{2}\left|\tau_{k, t} * S_{j+k} f(x)\right|^{2} d t\right)^{1 / 2} .
$$

Invoking Lemma 2.1 and the Littlewood-Paley theory imply

$$
\left\|I_{j} f\right\|_{p} \leq C\left\|\left(\sum_{k \in \mathbb{Z}}\left|S_{j+k} f\right|^{2}\right)^{1 / 2}\right\|_{p} \leq C\|f\|_{p}, \quad \forall 1<p<\infty
$$

On the other hand, by Plancherel's theorem and (2.4)-(2.5), we have

$$
\begin{aligned}
\left\|I_{j} f\right\|_{2}^{2} & =\int_{1}^{2} \sum_{k \in \mathbb{Z}} \int_{\mathbb{R}^{n}}|\hat{f}(\xi)|^{2}\left|\lambda_{j+k}(|L(\xi)|)\right|^{2}\left|\widehat{\tau_{k, t}}(\xi)\right|^{2} d \xi d t \\
& \leq C \int_{1}^{2} \sum_{k \in \mathbb{Z}} \int_{\left\{a_{j+k+1}^{-1} \leq|L(\xi)| \leq a_{j+k-1}^{-1}\right\}}|\hat{f}(\xi)|^{2}\left|\widehat{\tau_{k, t}}(\xi)\right|^{2} d \xi d t \\
& \leq C B_{j}^{2}\|f\|_{2}^{2},
\end{aligned}
$$

where $B_{j}=a^{-j+1} \chi_{\{j \geq-1\}}+(|j+1| \log a)^{-\beta} \chi_{\{j<-1\}}$. That is,

$$
\left\|I_{j} f\right\|_{2} \leq C B_{j}\|f\|_{2}
$$

Interpolating between (2.9) and (2.10), there exists $\epsilon \in(2 /(2 \beta+1), 1)$ such that

$$
\left\|I_{j} f\right\|_{p} \leq C B_{j}^{\epsilon}\|f\|_{p}, \quad \forall 1+1 /(2 \beta)<p<2 .
$$

For fixed $p \in(1+1 /(2 \beta), 2)$ and $\beta>1 / 2$, we can choose $q \in(1, p)$ such that $q \epsilon \beta>1$. Thus

$$
\sum_{j \in \mathbb{Z}}\left\|I_{j} f\right\|_{p}^{q} \leq C\left(\sum_{j \geq-1} a^{-q \epsilon(j-1)}+\sum_{j<-1}(|j+1| \log a)^{-q \epsilon \beta}\right)\|f\|_{p}^{q} \leq C(a)\|f\|_{p}^{q},
$$


which, together with (2.8), implies

$$
\|G(f)\|_{p} \leq C(a)\|f\|_{p}, \quad \text { for } 1+1 /(2 \beta)<p<2 .
$$

Case 2. $2<p<1+2 \beta$. By (2.3) and (2.7), we have for $2<p<\infty$ and $1<q<p^{\prime}$,

$$
\|G(f)\|_{p}^{q} \leq C \sum_{j \in \mathbb{Z}}\left(\int_{1}^{2}\left\|\left(\sum_{k \in \mathbb{Z}}\left|\tau_{k, t} * S_{j+k} f\right|^{2}\right)^{1 / 2}\right\|_{p}^{2} d t\right)^{q / 2} .
$$

Let

$$
J_{j, t} f(x):=\left(\sum_{k \in \mathbb{Z}}\left|\tau_{k, t} * S_{j+k} f(x)\right|^{2}\right)^{1 / 2}
$$

By (2.6), [23, p.544, Lemma] and the Littlewood-Paley theory, we have, for $k \in \mathbb{Z}$ and $t \in[1,2]$,

$$
\left\|J_{j, t} f\right\|_{p_{0}} \leq C\left\|\left(\sum_{k \in \mathbb{Z}}\left|S_{j+k} f\right|^{2}\right)^{1 / 2}\right\|_{p_{0}} \leq C\|f\|_{p_{0}}, \quad \forall 1<p_{0}<\infty
$$

On the other hand, by the same arguments as in (2.10), we have

$$
\left\|J_{j, t} f\right\|_{2} \leq C B_{j}\|f\|_{2},
$$

where $B_{j}$ is as in (2.10). On interpolation between (2.13) and (2.14), for fixed $p \in(2,1+2 \beta)$ and $\beta>1 / 2$, we can choose $q \in\left(1, p^{\prime}\right)$ and $\delta \in(2 /(2 \beta+1), 1)$ such that $q \delta \beta>1$ and

$$
\left\|J_{j, t} f\right\|_{p} \leq C B_{j}^{\delta}\|f\|_{p}, \quad \text { for } 2<p<1+2 \beta .
$$

This, combined with (2.12), implies

$$
\|G(f)\|_{p}^{q} \leq C\left(\sum_{j \geq-1} a^{-q \delta(j-1)}+\sum_{j<-1}(|j+1| \log a)^{-q \delta \beta}\right)\|f\|_{p}^{q} \leq C(a)\|f\|_{p}^{q},
$$

which, together with (2.11), completes the proof of Lemma 2.3.

Lemma 2.4 ([13, Lemma 2.2]) Suppose $\Phi(t)=t^{\alpha_{1}}+\mu_{2} t^{\alpha_{2}}+\cdots+\mu_{n} t^{\alpha_{n}}$ and $\varphi \in \mathfrak{F}$, where $\mu_{2}, \ldots, \mu_{n}$ are real parameters, and $\alpha_{1}, \ldots, \alpha_{n}$ are distinct positive (not necessarily integer) exponents. Then for any $r>0$ and $\lambda \in \mathbb{R} \backslash\{0\}$,

$$
\left|\int_{r / 2}^{r} \exp (i \lambda \Phi(\varphi(t))) \frac{d t}{t}\right| \leq C(\varphi)\left|\lambda \varphi(r)^{\alpha_{1}}\right|^{-\epsilon},
$$

where $\epsilon=\min \left\{1 / \alpha_{1}, 1 / n\right\}$ and $C(\varphi)$ does not depend on $\mu_{2}, \ldots, \mu_{n}$.

Lemma $2.5\left(\left[24\right.\right.$, Lemma 2.2]) Let $\mathcal{P}(t)=\left(P_{1}(t), \ldots, P_{d}(t)\right)$ with $P_{j}$ being real polynomials defined on $\mathbb{R}^{+}$. Suppose that $\varphi \in \mathfrak{F}$. Then the operator $M_{\mathcal{P}, \varphi}$ defined by

$$
M_{\mathcal{P}, \varphi}(f)(x)=\sup _{r>0} \int_{r}^{2 r}|f(x-\mathcal{P}(\varphi(t)))| \frac{d t}{t}
$$


is bounded on $L^{p}\left(\mathbb{R}^{d}\right)$ for $1<p<\infty$. The bound is independent of the coefficients of $P_{j}$ for all $1 \leq j \leq d$ and $f$ but depends on $\varphi$.

Lemma 2.6 ([25]) Let $\Phi: S^{1} \rightarrow \mathbb{R}^{d}, \Phi=\left(\Phi_{1}, \ldots, \Phi_{d}\right)$ be real analytic on $S^{1}$. Suppose that $\left\{\Phi_{1}, \ldots, \Phi_{d}\right\}$ is linearly independent set. If $\Omega \in \mathcal{F}_{\beta}\left(S^{1}\right)$ for some $\beta>1$, then

$$
\sup _{\xi \in S^{d-1}} \int_{S^{1}}\left|\Omega\left(y^{\prime}\right)\right|\left(\log ^{+} \frac{1}{\left|\xi \cdot \Phi\left(y^{\prime}\right)\right|}\right)^{\beta} d \sigma\left(y^{\prime}\right)<\infty
$$

\section{Proofs of main theorems}

Proof of Theorem 1.1 Let $\mathcal{N}=\max _{1 \leq j \leq n} \operatorname{deg}\left(P_{j}\right)$. For $1 \leq l \leq n$, let $P_{l}(t)=\sum_{i=1}^{\mathcal{N}} a_{i, l} t^{i}$. For $1 \leq$ $s \leq \mathcal{N}$, and $1 \leq l \leq n$, let $P_{l}^{(s)}(t)=\sum_{i=1}^{s} a_{i, l} t^{i}$ and $P^{(s)}(t)=\left(P_{1}^{(s)}(t), \ldots, P_{n}^{(s)}(t)\right)$. Set $P^{(0)}(t)=0$ and

$$
\Phi_{s}(y)=\left(P_{1}^{(s)}(\varphi(\rho(y))) y_{1}^{\prime}, \ldots, P_{n}^{(s)}(\varphi(\rho(y))) y_{n}^{\prime}\right) .
$$

Then we can write

$$
\begin{aligned}
\Phi_{s}(y) \cdot \xi & =\sum_{l=1}^{n} \xi_{l} y_{l}^{\prime} P_{l}^{(s)}(\varphi(\rho(y))) \\
& =\sum_{l=1}^{n} \sum_{i=1}^{s} \xi_{l} y_{l}^{\prime} a_{i, l} \varphi(\rho(y))^{i}=\sum_{i=1}^{s}\left(L_{i}(\xi) \cdot y^{\prime}\right) \varphi(\rho(y))^{i},
\end{aligned}
$$

where $L_{i}: \mathbb{R}^{n} \rightarrow \mathbb{R}^{n}$ is the linear transformation given by

$$
L_{i}(\xi)=\left(a_{i, 1} \xi_{1}, \ldots, a_{i, n} \xi_{n}\right)
$$

For each $j \in \mathbb{Z}, t \in \mathbb{R}^{+}$and $1 \leq s \leq \mathcal{N}$, we define the measures $\left\{\sigma_{j, t}^{s}\right\}$ and $\left\{\left|\sigma_{j, t}^{s}\right|\right\}$ by

$$
\begin{aligned}
& \widehat{\sigma_{j, t}^{s}}(\xi)=\frac{1}{2 i t} \int_{2^{j-1} t<\rho(y) \leq 2 j t} \exp \left(-2 \pi i \Phi_{s}(y) \cdot \xi\right) \frac{\Omega(y)}{\rho(y)^{\alpha-1}} d y, \\
& \widehat{\mid \sigma_{j, t}^{s}} \mid(\xi)=\frac{1}{2 i t} \int_{2^{j-1} t<\rho(y) \leq 2 j t} \exp \left(-2 \pi i \Phi_{s}(y) \cdot \xi\right) \frac{|\Omega(y)|}{\rho(y)^{\alpha-1}} d y .
\end{aligned}
$$

We get from (3.1)

$$
\begin{aligned}
& \left|\widehat{\sigma_{j, t}^{s}}(\xi)-\widehat{\sigma_{j, t}^{s-1}}(\xi)\right| \\
& \quad \leq \frac{1}{2 j} \int_{2^{j-1} t<\rho(y) \leq 2^{j} t}\left|\exp \left(-2 \pi i \Phi_{s}(y) \cdot \xi\right)-\exp \left(-2 \pi i \Phi_{s-1}(y) \cdot \xi\right)\right| \frac{|\Omega(y)|}{\rho(y)^{\alpha-1}} d y \\
& \quad \leq C\left(\varphi\left(2^{j} t\right)^{s}\left|L_{s}(\xi)\right|\right) .
\end{aligned}
$$


On the other hand, by a change of variable, we have

$$
\begin{aligned}
\left|\widehat{\sigma_{j, t}^{s}}(\xi)\right| & =\frac{1}{2^{j} t}\left|\int_{2^{j-1} t}^{2^{j} t} \int_{S^{n-1}} \exp \left(-2 \pi i \sum_{k=1}^{s} L_{k}(\xi) \cdot \theta \varphi(r)^{k}\right) \Omega(\theta) J(\theta) d \sigma(\theta) d r\right| \\
& \leq C \int_{S^{n-1}}|\Omega(\theta)|\left|I_{j, t, s, \xi}(\theta)\right| d \sigma(\theta),
\end{aligned}
$$

where

$$
I_{j, t, s, \xi}(\theta):=\frac{1}{2^{j} t} \int_{2^{j-1} t}^{2^{j} t} \exp \left(-2 \pi i \sum_{k=1}^{s} L_{k}(\xi) \cdot \theta \varphi(r)^{k}\right) d r .
$$

By Lemma 2.4, we have

$$
\left|I_{j, t, s, \xi}(\theta)\right| \leq C\left|\varphi\left(2^{j} t\right)^{s} L_{s}(\xi) \cdot \theta\right|^{-1 / s}
$$

Combining the trivial inequality $\left|I_{j, t, s, \xi}(\theta)\right| \leq C$ with the fact that $t /(\log t)^{\beta}$ is increasing in $\left(e^{\beta}, \infty\right)$, we have

$$
\left|I_{j, t, s, \xi}(\theta)\right| \leq C \frac{\left(\log e^{\beta s}|\eta \cdot \theta|^{-1}\right)^{\beta}}{\left(\log \left|\varphi\left(2^{j} t\right)^{s} L_{s}(\xi)\right|\right)^{\beta}}, \quad \text { if }\left|\varphi\left(2^{j} t\right)^{s} L_{s}(\xi)\right|>1
$$

where $\eta=L_{s}(\xi) /\left|L_{s}(\xi)\right|$. This, together (3.3) with the fact that $\Omega \in \mathcal{F}_{\beta}\left(S^{n-1}\right)$, implies

$$
\left|\widehat{\sigma_{j, t}^{s}}(\xi)\right| \leq C\left(\log \left|\varphi\left(2^{j} t\right)^{s} L_{s}(\xi)\right|\right)^{-\beta}, \quad \text { if }\left|\varphi\left(2^{j} t\right)^{s} L_{s}(\xi)\right|>1
$$

Now we can choose a function $\psi \in C_{0}^{\infty}(\mathbb{R})$ such that $\psi(t) \equiv 1$ for $|t| \leq 1 / 2$ and $\psi(t) \equiv 0$ for $|t|>1$. For $1 \leq s \leq \mathcal{N}, j \in \mathbb{Z}$ and $t \in \mathbb{R}^{+}$, we define the measures $\left\{\tau_{j, t}^{s}\right\}$ by

$$
\begin{aligned}
\widehat{\tau_{j, t}^{s}}(\xi)= & \widehat{\sigma_{j, t}^{s}}(\xi) \prod_{k=s+1}^{\mathcal{N}} \psi\left(\left|\varphi\left(2^{j} t\right)^{k} L_{k}(\xi)\right|\right) \\
& -\widehat{\sigma_{j, t}^{s-1}}(\xi) \prod_{k=s}^{\mathcal{N}} \psi\left(\left|\varphi\left(2^{j} t\right)^{k} L_{k}(\xi)\right|\right) .
\end{aligned}
$$

Here we use the convention $\prod_{j \in \emptyset} a_{j}=1$. It is easy to see that

$$
\sigma_{j, t}^{\mathcal{N}}=\sum_{s=1}^{\mathcal{N}} \tau_{j, t}^{s}
$$

It follows from (3.2), (3.5), and the trivial estimate $\left|\widehat{\sigma_{j, t}^{s}}(\xi)\right| \leq C$ that, for $1 \leq s \leq \mathcal{N}$,

$$
\begin{aligned}
& \left|\widehat{\tau_{j, t}^{s}}(\xi)\right| \leq C(\varphi) \min \left\{1, \varphi\left(2^{j} t\right)^{s}\left|L_{s}(\xi)\right|\right\}, \\
& \left|\widehat{\tau_{j, t}^{s}}(\xi)\right| \leq C(\varphi)\left(\log \left|\varphi\left(2^{j} t\right)^{s} L_{s}(\xi)\right|\right)^{-\beta}, \quad \text { if }\left|\varphi\left(2^{j} t\right)^{s} L_{s}(\xi)\right|>1 .
\end{aligned}
$$


By the definition of $\sigma_{j, t}^{s}$ and (3.6), we can write

$$
\begin{aligned}
\mathcal{M}_{\Omega}^{\Phi}(f)(x) & =\left(\int_{0}^{\infty}\left|\sum_{j=-\infty}^{0} 2^{j} \sigma_{j, t}^{\mathcal{N}} * f(x)\right|^{2} \frac{d t}{t}\right)^{1 / 2} \\
& \leq \sum_{j=-\infty}^{0} 2^{j}\left(\int_{0}^{\infty}\left|\sigma_{j, t}^{\mathcal{N}} * f(x)\right|^{2} \frac{d t}{t}\right)^{1 / 2} \\
& \leq 2\left(\sum_{j \in \mathbb{Z}} \int_{2^{j}}^{2^{j+1}}\left|\sigma_{0, t}^{\mathcal{N}} * f(x)\right|^{2} \frac{d t}{t}\right)^{1 / 2} \\
& \leq 2\left(\sum_{j \in \mathbb{Z}} \int_{1}^{2}\left|\sigma_{j, t}^{\mathcal{N}} * f(x)\right|^{2} \frac{d t}{t}\right)^{1 / 2} \\
& \leq 2 \sum_{s=1}^{\mathcal{N}}\left(\int_{1}^{2} \sum_{j \in \mathbb{Z}}\left|\tau_{j, t}^{s} * f(x)\right|^{2} \frac{d t}{t}\right)^{1 / 2} .
\end{aligned}
$$

On the other hand, by a change of variable we have

$$
\begin{aligned}
|| \sigma_{j, t}^{s}|* f(x)| & \leq \frac{1}{2 j t} \int_{2^{j-1} t<\rho(y) \leq 2 j t}\left|f\left(x-\Phi_{s}(y)\right)\right| \frac{|\Omega(y)|}{\rho(y)^{\alpha-1}} d y \\
& \leq C \int_{S^{n-1}}\left|\Omega\left(y^{\prime}\right)\right|\left(\frac{1}{2^{j t}} \int_{2^{j-1} t}^{2^{j} t}\left|f\left(x-\Phi_{s}\left(A_{r} y^{\prime}\right)\right)\right| d r\right) d \sigma\left(y^{\prime}\right) \\
& \leq C \int_{S^{n-1}}\left|\Omega\left(y^{\prime}\right)\right| M_{\mathcal{P}, \varphi}(f)(x) d \sigma\left(y^{\prime}\right),
\end{aligned}
$$

where $M_{\mathcal{P}, \varphi}$ is as in Lemma 2.5 and $\mathcal{P}(t)=\left(P_{1}^{(s)}(t) y_{1}^{\prime}, \ldots, P_{n}^{(s)}(t) y_{n}^{\prime}\right)$. By Lemma 2.5 and Minkowski's inequality, we have

$$
\left\|\sup _{j \in \mathbb{Z}} \sup _{t>0}\right\| \sigma_{j, t}^{s}|* f|\left\|_{p} \leq C\right\| f \|_{p}
$$

This inequality, together with the definition of $\tau_{j, t}^{s}$, yields

$$
\left\|\sup _{j \in \mathbb{Z}} \sup _{t>0}|| \tau_{j, t}^{s}|* f|\right\|_{p} \leq C\|f\|_{p}
$$

Then Theorem 1.1 follows from (3.7)-(3.10) and Lemma 2.3.

Proof of Theorem 1.3 Let $\Phi, P_{N}, \varphi, \phi$ be as in Theorem 1.3. For $1 \leq s \leq N$, we set $P_{s}(t)=$ $\sum_{m=1}^{s} a_{m} t^{m}$. Define the measures $\left\{\sigma_{j, t}^{s}\right\}$ and $\left\{\left|\sigma_{j, t}^{s}\right|\right\}$ by

$$
\begin{aligned}
& \widehat{\sigma_{j, t}^{s}}(\xi)=\frac{1}{2 j t} \int_{2^{j-1} t<\rho(y) \leq 2 j_{t}} \exp \left(-2 \pi i P_{s}(\varphi(\rho(y))) \phi\left(y^{\prime}\right) \cdot \xi\right) \frac{\Omega(y)}{\rho(y)^{\alpha-1}} d y \\
& \widehat{\mid \sigma_{j, t}^{s}}(\xi)=\frac{1}{2 j t} \int_{2^{j-1} t<\rho(y) \leq 2 j_{t}} \exp \left(-2 \pi i P_{s}(\varphi(\rho(y))) \phi\left(y^{\prime}\right) \cdot \xi\right) \frac{|\Omega(y)|}{\rho(y)^{\alpha-1}} d y .
\end{aligned}
$$

Following the notation in [20], let $\left\{\phi_{i_{1}}, \ldots, \phi_{i_{l}}\right\}$ be a maximal linearly independent subset of $\left\{\phi_{1}, \ldots, \phi_{d}\right\}$, where $1 \leq l \leq d, 1 \leq i_{r} \leq d$ and $r=1, \ldots, l$. Thus, for $j \notin\left\{i_{1}, \ldots, i_{l}\right\}$, there exist 
$a^{(j)}=\left(a_{j, 1}, \ldots, a_{j, l}\right) \in \mathbb{R}^{l}$ such that

$$
\phi_{j}\left(y^{\prime}\right)=a^{(j)} \cdot\left(\phi_{i_{1}}\left(y^{\prime}\right), \ldots, \phi_{i_{l}}\left(y^{\prime}\right)\right)=\sum_{k=1}^{l} a_{j, k} \phi_{i_{k}}\left(y^{\prime}\right) .
$$

This implies that there exists a linear transformation $L: \mathbb{R}^{d} \rightarrow \mathbb{R}^{l}$ such that

$$
\phi\left(y^{\prime}\right) \cdot \xi=L(\xi) \cdot \tilde{\phi}\left(y^{\prime}\right), \quad \xi \in \mathbb{R}^{d},
$$

where $\tilde{\phi}\left(y^{\prime}\right)=\left(\phi_{i_{1}}\left(y^{\prime}\right), \ldots, \phi_{i_{l}}\left(y^{\prime}\right)\right)$. Thus

$$
\left|\widehat{\sigma_{j, t}^{s}}(\xi)\right| \leq C \int_{S^{1}}\left|\Omega\left(z^{\prime}\right)\right|\left|I_{j, t, s, \xi}\left(z^{\prime}\right)\right| d \sigma\left(z^{\prime}\right)
$$

where

$$
I_{j, t, s, \xi}\left(z^{\prime}\right):=\frac{1}{2^{j} t} \int_{2^{j-1} t}^{2^{j} t} \exp \left[-2 \pi i P_{s}(\varphi(u))\left(L(\xi) \cdot \tilde{\phi}\left(z^{\prime}\right)\right)\right] d u .
$$

By Lemma 2.4, we have

$$
\left|I_{j, t, s, \xi}\left(z^{\prime}\right)\right| \leq C\left|\varphi\left(2^{j} t\right)^{s} a_{s} L(\xi) \cdot \tilde{\phi}\left(z^{\prime}\right)\right|^{-1 / s} .
$$

Since $t /(\log t)^{\beta}$ is increasing in $\left(e^{\beta}, \infty\right)$ for any $\beta>0$, and $\left|\tilde{\phi}\left(z^{\prime}\right)\right| \leq B$ with $B>1$ for any $z^{\prime} \in S^{n-1}$. We can deduce from (3.13) and the trivial estimate $\left|I_{j, t, s, \xi}\left(z^{\prime}\right)\right| \leq C$ that

$$
\left|I_{j, t, s, \xi}\left(z^{\prime}\right)\right| \leq C \frac{\left(\log \left(B e^{\beta s}\left|\zeta \cdot \tilde{\phi}\left(z^{\prime}\right)\right|^{-1}\right)\right)^{\beta}}{\left(\log \left|\varphi\left(2^{j} t\right)^{s} a_{s} L(\xi)\right|\right)^{\beta}}, \quad \text { if }\left|\varphi\left(2^{j} t\right) a_{s} L(\xi)\right|>1
$$

where $\zeta=L(\xi) /|L(\xi)|$. Invoking Lemma 2.6 and (3.14), we obtain, for $\beta>1$,

$$
\left|\widehat{\sigma_{j, t}^{s}}(\xi)\right| \leq C\left(\log \left|\varphi\left(2^{j} t\right)^{s} a_{s} L(\xi)\right|\right)^{-\beta}, \quad \text { if }\left|\varphi\left(2^{j} t\right)^{s} a_{s} L(\xi)\right|>1
$$

On the other hand, we have

$$
\begin{aligned}
\widehat{\sigma_{j, t}^{s}}(\xi)-\widehat{\sigma_{j, t}^{s-1}}(\xi) \mid & \leq \frac{1}{2^{j} t} \int_{2^{j-1} t<\rho(y) \leq 2^{j} t}\left|\exp \left(-2 \pi i\left(a_{s} \varphi(\rho(y))^{s}|L(\xi)| \tilde{\phi}\left(y^{\prime}\right)\right)\right)-1\right| \frac{|\Omega(y)|}{\rho(y)^{\alpha-1}} d y \\
& \leq C\left|\varphi\left(2^{j} t\right)^{s} a_{s} L(\xi)\right| \int_{S^{1}}\left|\Omega\left(z^{\prime}\right)\right|\left|\tilde{\phi}\left(z^{\prime}\right)\right| d \sigma\left(z^{\prime}\right) \\
& \leq C\left|\varphi\left(2^{j} t\right)^{s} a_{s} L(\xi)\right| \sup _{z^{\prime} \in S^{1}}\left|\tilde{\phi}\left(z^{\prime}\right)\right| \\
& \leq C\left|\varphi\left(2^{j} t\right)^{s} a_{s} L(\xi)\right| .
\end{aligned}
$$

Notice that

$$
\begin{aligned}
|| \sigma_{j, t}^{s}|* f(x)| & \leq \frac{1}{2 j t} \int_{2^{j-1} t<\rho(y) \leq 2 j t}\left|f\left(x-P_{s}(\varphi(\rho(y))) \phi\left(y^{\prime}\right)\right)\right| \frac{|\Omega(y)|}{\rho(y)^{\alpha-1}} d y \\
& \leq C \int_{S^{1}}\left|\Omega\left(y^{\prime}\right)\right|\left(\int_{0}^{2^{2} t}\left|f\left(x-P_{s}(\varphi(u)) \phi\left(y^{\prime}\right)\right)\right| \frac{d u}{u}\right) d \sigma\left(y^{\prime}\right) .
\end{aligned}
$$


This combining with Lemma 2.5 and Minkowski's inequality, implies

$$
\left\|\sup _{j \in \mathbb{Z}} \sup _{t>0}|| \sigma_{j, t}^{s}|* f|\right\|_{p} \leq C\|f\|_{p}
$$

Then the rest of the proof of Theorem 1.3 follows from an argument which is similar to those in the proof of Theorem 1.1 and (3.15)-(3.17). We omit the details.

Proof of Theorem 1.4 Let $n \geq 2, \mathcal{P}=\left(P_{1}, \ldots, P_{d}\right)$, where $P_{j}: \mathbb{R}^{n} \rightarrow \mathbb{R}$ is a polynomial for $1 \leq j \leq d$. Let

$$
M=\max \left\{\operatorname{deg}\left(P_{1}\right), \ldots, \operatorname{deg}\left(P_{d}\right)\right\},
$$

and

$$
P_{i}(y)=\sum_{|\gamma| \leq M} a_{i \gamma} y^{\gamma} \quad \text { for } i=1, \ldots, d .
$$

For $1 \leq s \leq M$, we let

$$
\mathcal{P}^{(s)}=\left(P_{1, s}, \ldots, P_{d, s}\right),
$$

where

$$
P_{i, s}(y)=\sum_{|\gamma| \leq s} a_{i \gamma} y^{\gamma}
$$

Set $\mathcal{P}^{(0)}=0$ and $\Phi_{s}(y)=\mathcal{P}^{(s)}\left(\varphi(\rho(y)) y^{\prime}\right)$.

For each $j \in \mathbb{Z}, t \in \mathbb{R}^{+}$and $0 \leq s \leq M$, we define the measures $\left\{\sigma_{j, t}^{s}\right\}$ and $\left\{\left|\sigma_{j, t}^{s}\right|\right\}$ by

$$
\begin{aligned}
& \widehat{\sigma_{j, t}^{s}}(\xi)=\frac{1}{2 j t} \int_{2^{j-1} t<\rho(y) \leq 2 j_{t}} \exp \left(-2 \pi i \Phi_{s}(y) \cdot \xi\right) \frac{\Omega(y)}{\rho(y)^{\alpha-1}} d y \\
& \widehat{\mid \sigma_{j, t}^{s}} \mid(\xi)=\frac{1}{2 j t} \int_{2^{j-1} t<\rho(y) \leq 2 j_{t}} \exp \left(-2 \pi i \Phi_{s}(y) \cdot \xi\right) \frac{|\Omega(y)|}{\rho(y)^{\alpha-1}} d y .
\end{aligned}
$$

For $1 \leq s \leq M$, let $l_{s}$ denote the number of multi-indices $\gamma=\left(\gamma_{1}, \ldots, \gamma_{n}\right)$ satisfying $|\gamma|=s$, and define the linear transformation $L_{s}: \mathbb{R}^{d} \rightarrow \mathbb{R}^{l_{s}}$ by

$$
L_{s}(\xi)=\left(\left(L_{s}(\xi)\right)_{\gamma}\right)_{|\gamma|=s}=\left(\sum_{i=1}^{d} a_{i \gamma} \xi_{i}\right)_{|\gamma|=s} .
$$

By the change of variables, we have

$$
\begin{aligned}
\left|\widehat{\sigma_{j, t}^{s}}(\xi)\right| & =\left|\int_{S^{n-1}} \frac{1}{2^{j} t} \int_{2^{j-1} t}^{2^{j} t} \exp \left(-2 \pi i \xi \cdot \mathcal{P}^{(s)}(\varphi(u) \theta)\right) d u \Omega(\theta) J(\theta) d \sigma(\theta)\right| \\
& \leq C \int_{S^{n-1}}|\Omega(\theta)|\left|\frac{1}{2^{j} t} \int_{2^{j-1} t}^{2^{j} t} \exp \left(-2 \pi i \xi \cdot \mathcal{P}^{(s)}(\varphi(u) \theta)\right) d u\right| d \sigma(\theta)
\end{aligned}
$$




$$
\begin{aligned}
& \leq C \int_{S^{n-1}}|\Omega(\theta)|\left|\frac{1}{2 j t} \int_{2^{j-1} t}^{2^{j} t} \exp \left(-2 \pi i \sum_{|\gamma| \leq s} \sum_{i=1}^{d} \xi_{j} a_{j \gamma} \theta^{\gamma} \varphi(u)^{|\gamma|}\right) d u\right| d \sigma(\theta) \\
& \leq C \int_{S^{n-1}}|\Omega(\theta)|\left|j_{j, t, s, \xi}(\theta)\right| d \sigma(\theta)
\end{aligned}
$$

where

$$
J_{j, t, s, \xi}(\theta):=\frac{1}{2 i t} \int_{2^{j-1} t}^{2 j t} \exp \left(-2 \pi i \sum_{|\gamma|=s}\left(L_{s}(\xi)\right) \gamma \theta^{\gamma} \varphi(u)^{s}+\text { lower powers in } u\right) d u \text {. }
$$

Let

$$
Q_{s, \xi}(\theta):=\left|L_{s}(\xi)\right|^{-1} \sum_{|\gamma|=s}\left(L_{s}(\xi)\right) \gamma \theta^{\gamma}
$$

By Lemma 2.4, we have

$$
\left|J_{j, t, s, \xi}(\theta)\right| \leq C\left(\varphi\left(2^{j} t\right)^{s}\left|L_{s}(\xi)\right|\left|Q_{s, \xi}(\theta)\right|\right)^{-1 / s}
$$

By this inequality, together with the trivial estimate $\left|J_{j, t, s, \xi}(\theta)\right| \leq C$, we get

$$
\left|J_{j, t, s, \theta}(\xi)\right| \leq C \frac{\left(\log \left(e^{\beta s} l_{s}^{1 / 2}\left|Q_{s, \xi}(\theta)\right|^{-1}\right)\right)^{\beta}}{\left(\log \left|\varphi\left(2^{j} t\right)^{s} L_{s}(\xi)\right|\right)^{\beta}}, \quad \text { if }\left|\varphi\left(2^{j} t\right)^{s} L_{s}(\xi)\right|>1 .
$$

Since $\Omega \in \bigcap_{s=1}^{\infty} \mathcal{F}(n, s, \beta), Q_{s, \xi} \in V(n, s)$ and $\left\|Q_{s, \xi}\right\|=1$, we immediately obtain

$$
\left|\widehat{\sigma_{j, t}^{s}}(\xi)\right| \leq C\left(\log \left|\varphi\left(2^{j} t\right)^{s} L_{s}(\xi)\right|\right)^{-\beta}, \quad \text { if }\left|\varphi\left(2^{j} t\right)^{s} L_{s}(\xi)\right|>1 .
$$

On the other hand, we have

$$
\begin{aligned}
& \left|\widehat{\sigma_{j, t}^{s}}(\xi)-\widehat{\sigma_{j, t}^{s-1}}(\xi)\right| \\
& \quad \leq \frac{1}{2^{j t}} \int_{2^{j-1} t<\rho(y) \leq 2 t}\left|\exp \left(-2 \pi i \sum_{j=1}^{d} \sum_{|l|=s} \xi_{j} a_{j l} \varphi(\rho(y))^{s}\left(y^{\prime}\right)^{\iota}\right)-1\right| \frac{|\Omega(y)|}{\rho(y)^{\alpha-1}} d y \\
& \quad \leq C\left|\varphi\left(2^{j} t\right)^{s} L_{s}(\xi)\right| .
\end{aligned}
$$

In addition, using Lemma 2.5 , one can easily check that

$$
\left\|\sup _{j \in \mathbb{Z}} \sup _{t>0}|| \sigma_{j, t}^{s}|* f|\right\|_{p} \leq C\|f\|_{p}
$$

Then the rest proof of Theorem 1.4 follows from similar arguments to the proof of Theorem 1.1 and (3.18)-(3.20). Details will be omitted. 
Authors' contributions

The authors worked jointly in drafting and approving the final manuscript.

\section{Author details}

'School of Mathematical Sciences, Xiamen University, Xiamen, 361005, China. ${ }^{2}$ College of Mathematics and Systems Science, Shandong University of Science and Technology, Qingdao, Shandong 266590, China.

\section{Acknowledgements}

The authors would like to thank the referees for their carefully reading and invaluable comments. This work was supported by the NNSF of China (Nos. 11101339, 11371295).

Received: 23 February 2014 Accepted: 30 June 2014 Published: 22 Jul 2014

\section{References}

1. Fabes, E, Rivière, N: Singular integrals with mixed homogeneity. Stud. Math. 27, 19-38 (1966)

2. Stein, EM: On the function of Littlewood-Paley, Lusin and Marcinkiewicz. Trans. Am. Math. Soc. 88, $430-466$ (1958)

3. Benedek, A, Calderón, A, Panzone, R: Convolution operators on Banach space valued functions. Proc. Natl. Acad. Sci. USA 48, 356-365 (1962)

4. Chen, J, Fan, D, Pan, Y: A note on a Marcinkiewicz integral operator. Math. Nachr. 227, 33-42 (2001)

5. Ding, $Y$, Fan, $D$, Pan, $Y: L^{P}$-boundedness of Marcinkiewicz integrals with Hardy space function kernel. Acta Math. Sin. Engl. Ser. 16, 593-600 (2000)

6. Ding, $Y$, Pan, $Y: L^{P}$ bounds for Marcinkiewicz integrals. Proc. Edinb. Math. Soc. 46, 669-677 (2003)

7. $\mathrm{Ma}, \mathrm{B}, \mathrm{Wu}, \mathrm{H}, \mathrm{Zhao}, \mathrm{X}$ : Rough Marcinkiewicz integrals along certain smooth curves. Front. Math. China 7(5), 857-872 (2012)

8. $\mathrm{Wu}, \mathrm{H}: L^{p}$ bounds for Marcinkiewicz integrals associates to surfaces of revolution. J. Math. Anal. Appl. 321, 811-827 (2006)

9. $\mathrm{Xu}, \mathrm{H}$, Chen, J, Ying, Y: A note on Marcinkiewicz integrals with $H^{1}$ kernels. Acta Math. Sci. 23(1), 133-138 (2003)

10. Grafakos, L, Stefanov, A: $L^{p}$ bounds for singular integrals and maximal singular integrals with rough kernels. Indiana Univ. Math. J. 47, 455-469 (1998)

11. Al-Salman, A, Al-Qassem, H, Cheng, LC, Pan, Y: $L^{p}$ bounds for the function of Marcinkiewicz. Math. Res. Lett. 9, 697-700 (2002)

12. Wu, H: On Marcinkiewicz integral operators with rough kernels. Integral Equ. Oper. Theory 52, $285-298$ (2005)

13. Liu, F, Wu, H, Zhang, D: Parametric Marcinkiewicz integrals with rough kernels supported by compound subvariaties. Acta. Math. Sci. (Chin. Ser.) (2014, in press)

14. Ding, Y, Xue, Q, Yabuta, K: Parabolic Littlewood-Paley g-function with rough kernel. Acta Math. Sin. Engl. Ser. 24(10), 2049-2060 (2008)

15. Chen, $Y$, Ding, $Y: L^{p}$ bounds for parabolic Marcinkiewicz integral with rough kernels. J. Korean Math. Soc. 44, 733-745 (2007)

16. Chen, $D, L u, S: L^{p}$ boundedness of parabolic Littlewood-Paley operator with rough kernel belonging to $F_{\alpha}\left(\mathbb{S}^{n-1}\right)$. Acta Math. Sci. 31(2), 343-350 (2011)

17. Liu, F, Wu, H: Rough Marcinkiewicz integrals with mixed homogeneity on product spaces. Acta Math. Sin. Engl. Ser. 29(7), 1231-1244 (2013)

18. Al-Salman, A: A note on parabolic Marcinkiewicz integrals along surfaces. Proc. A. Razmadze Math. Inst. 154, 21-36 (2010)

19. Al-Salman, A: Parabolic Marcinkiewicz integrals along surfaces on product domains. Acta Math. Sin. Engl. Ser. 27(1), 1-18 (2011)

20. Al-Balushi, $\mathrm{K}, \mathrm{Al}-\mathrm{Salman}, \mathrm{A}$ : Certain $L^{p}$ bounds for rough singular integrals. Preprint (2013)

21. Al-Salman, A, Pan, Y: Singular integrals with rough kernels. Can. Math. Bull. 47(1), 3-11 (2004)

22. Al-Salman, A, Al-Qassem, H: Rough Marcinkiewicz integral operators. Int. J. Math. Math. Sci. 27(8), 495-503 (2001)

23. Duoandikoetxea, J, Rubio de Francia, JL: Maximal and singular integral operators via Fourier transform estimates. Invent. Math. 84, 541-561 (1986)

24. Liu, F, Wu, H: Multiple singular integrals and Marcinkiewicz integrals with mixed homogeneity along surfaces. J. Inequal. Appl. 2012(189), 1-23 (2012)

25. Al-Salman, A: Estimates of singular integral operators of convolution type with rough kernels. PhD Dissertation, University of Pittsburgh (1999)

10.1186/1029-242X-2014-265

Cite this article as: Zhang and Liu: Boundedness of Marcinkiewicz integrals with mixed homogeneity along compound surfaces. Journal of Inequalities and Applications 2014, 2014:265 